\title{
BMJ Open Cohort profile: the Welsh Geriatric Registrar-Led Research Network (WeGeN): rationale, design and description
}

\author{
Benjamin Jelley, ${ }^{1}$ Sara Long, ${ }^{2}$ John Butler, ${ }^{1}$ Jonathan Hewitt, ${ }^{3}$ on behalf of WeGeN
}

To cite: Jelley B, Long S, Butler J, et al. Cohort profile: the Welsh Geriatric RegistrarLed Research Network (WeGeN): rationale, design and description. BMJ Open 2017;7:e013031.

doi:10.1136/bmjopen-2016013031

- Prepublication history for this paper is available online. To view these files please visit the journal online (http://dx.doi.org/10.1136/ bmjopen-2016-013031).

Received 16 June 2016 Revised 8 November 2016 Accepted 22 December 2016

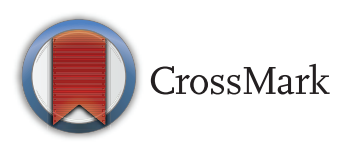

${ }^{1}$ Department of Gerontology, Cardiff and Vale University Health Board, Cardiff, UK

${ }^{2}$ Aneurin Bevan University Health Board, Newport, UK ${ }^{3}$ Department of Geriatric Medicine, Cardiff University, Cardiff, UK

Correspondence to Dr Jonathan Hewitt; hewittj2@cardiff.ac.uk

\section{ABSTRACT}

Purpose: Medical trainees are required to undertake audit and quality improvement projects. They must also have an understanding of the principles of research and are encouraged to participate in research projects. However, the constraints of time, a lack of formal training and rotation between different training posts create barriers to audit cycle completion and pursuing research. This leads to trainees being reluctant to undertake research, facilitates poor quality research and risks incomplete audit.

Participants: The Welsh Geriatricians Network (WeGeN) has been created with the aims of facilitating collaborative, trainee-led research within Geriatric Medicine in Wales, promoting research engagement and improving the research evidence base for older patients. By coordinating collaborative research projects across different sites within Wales, trainees continue existing projects at new sites, allowing completion of projects and establishing the long-term infrastructure and experienced personnel needed for high-quality research data to be gathered.

Findings: WeGeN has facilitated 4 national audits, all of which are intended for peer review publication. The first project considers the service provision for the older person in the emergency department, the second Parkinson's disease, the third reviews delirium management and the fourth project considers epidemiology of surgical disease in older people.

Future plans: The objective of this project is to further establish and develop WeGeN as a group which facilitates high-quality research and provides the opportunity for geriatric trainees to engage in research activity. It is anticipated that the establishment of this research platform will provide a blueprint for the development of other such networks in the UK and beyond.

\section{INTRODUCTION}

Geriatric Medicine Specialty Trainees are required to have an understanding of the principles of research and are encouraged to participate in research projects either during their training or as a period out of

\section{Strengths and limitations of this study}

- The opportunity for large-scale high-quality research exposure for geriatric trainees is the major strength of the WeGeN collaboration.

- WeGeN will provide research-experienced geriatricians, and results generated will improve the evidence base for geriatric medicine.

- Owing to the nature and type of study sites accessible to WeGeN, it is anticipated that data will be representative not only of Wales but the UK as a whole.

- Research carried out by WeGeN is pragmatic, flexible and cost-effective.

- Limitations of our methods include those inherent with all observational data, such as lack of comparator group and site-to-site variability.

programme Specialty Training Curriculum for Geriatric Medicine. ${ }^{1}$ Scientific, peerreviewed publication can be used as a discriminator for selection criteria for career progression. ${ }^{2}$ Furthermore, research engagement during training promotes future research engagement and long-term participation in research. ${ }^{3}$ However, the constraints of time, a lack of training in research methodology and rotation between different training posts can create barriers to research, a reluctance to undertake research and may produce poor quality research. ${ }^{4}$

To overcome these barriers, it has become commonplace for surgical trainees to form regional research collaboratives ${ }^{5}$ structured in a similar fashion to other collaboratives such as those used in the primary care setting ${ }^{6} 7$ The surgical trainee-led collaboratives have now expanded from regional, to national, European and international collaborations. ${ }^{8}$ They have achieved impressive results in the $\mathrm{UK}^{9}{ }^{10}$ and internationally. ${ }^{11}$ For example, a recent international collaboration investigating mortality following surgery in high, middle and low-income countries 
recruited 10745 patients by 1465 collaborators, across 357 centres in 58 countries. $^{11}$

To address the lack of similar collaboration in geriatric medicine, we have created the Welsh Geriatricians Network (WeGeN) with the aims of facilitating collaborative, trainee-led research within geriatric medicine in Wales, promoting longer term research engagement and ultimately improving the research evidence base for older patients.

The purpose of this manuscript is to define our research network, present our methods and describe future intentions. We aim to do so in a transparent and well-structured manner in keeping with the current drive to standardise process and improve quality in clinical research. ${ }^{12}{ }^{13}$ We also hope that the description of the research network will publicise and inspire its use.

\section{METHODS}

The purpose of WeGeN is to provide a centrally coordinated research network that can be accessed and used by clinicians within Wales. Potential collaborators are invited to bring project ideas to the WeGeN team through email correspondence or face-to-face discussion with any of the members of the team. The team will then provide assistance with the set-up and delivery of the project.

The team currently consists of one network lead, a senior research advisor and a deputy network lead. The structure is given in figure 1 . The separate roles within the hierarchy are described in more detail below.

\section{THE NETWORK LEADS}

The network leads are responsible for the overall running of the network but do not have responsibility for the delivery of all projects supported by WeGeN. Their role focuses on liaising with other clinicians to

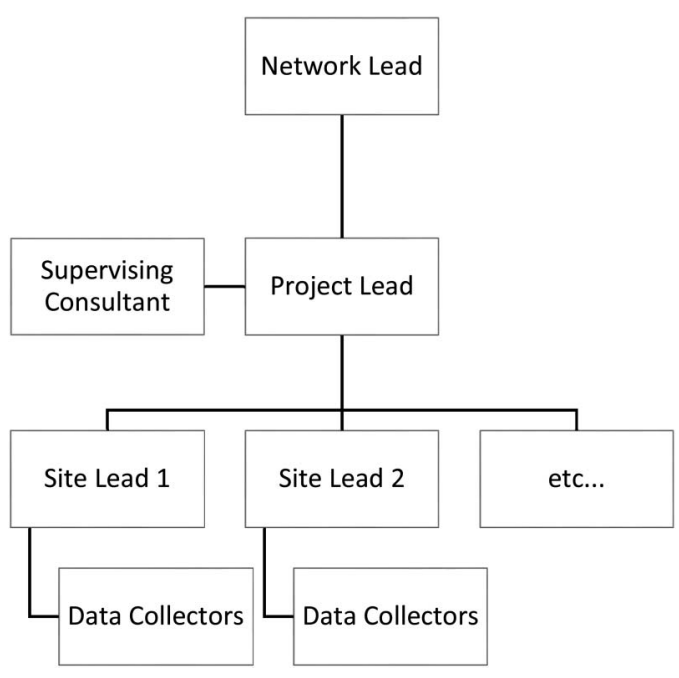

Figure 1 Hierarchy of personnel in network of Geriatrics Specialty Trainees. bring new projects to the Network and assisting with the initiation of projects by recruiting Specialty Trainees to participate. Close liaison with each project lead is also required to facilitate successful completion and ensure that the work is of high quality. All administrative work is currently undertaken by these two individuals.

\section{THE PROJECT LEAD}

This is the geriatrician who has brought the project to WeGeN or who volunteered to lead a group application. The project lead is responsible for the practical aspects of project delivery. Trainees leading projects will work with an engaged supervising consultant to establish a line of clinical responsibility. The project lead formulates the project's protocol and assists each site with initiation of the project.

The project lead is expected to obtain ethical approval. They are tasked with the ongoing delivery of the project including liaising with individual site leads to troubleshoot issues that may arise, and coordinating the multiple sites during the data collection period. Following completion of the project, the project lead will collate and analyse the data collected and coordinate the submission to peer-reviewed publications.

\section{THE SITE LEAD}

The site lead oversees data collection for a project at one site. That individual has responsibility to ensure successful completion of the project at that site under the guidance of a local clinician.

The site lead will liaise with local research and development, quality improvement or audit departments to ensure that the project is implemented and conducted according to local guidelines and protocols.

The site lead will educate the data collection team about project methodology and support them in data acquisition. Following the completion of data collection, the site lead will be able to analyse their locally collected data to give immediate local feedback, providing an opportunity to improve local performance and implement change.

To overcome the issue of trainee rotation impacting on project completion, WeGeN aims to work with the Geriatric Medicine Specialty Training Programme Director to attempt to anticipate where trainees will be placed. This will allow identification of sites where a data collection cycle can be successfully completed irrespective of clinical rotation. This will be achieved through doctors familiar with the site lead role rotating from one active project site to another, providing project continuity.

\section{THE DATA COLLECTORS}

Data collection will vary between project and study type. However, many projects will require the site lead to delegate and coordinate data collection between a team of 
people at their site. This could include students, junior doctors, specialist nursing staff and other members of the multidisciplinary team.

\section{Communication within the network}

Multicentre endeavours require regular communication with participants to ensure that potential problems can be addressed early and to maintain the motivation of the group.

Currently, Specialty Trainees in geriatric medicine in South Wales communicate through the team communication smartphone app Slack. ${ }^{14}$ This software allows rapid, nearly real-time communication with multiple people simultaneously. WeGeN recommend this medium or an agreed alternative to optimise project management by the project lead. Communication through traditional methods such as telephone and email is recommended to all project leads to increase accessibility and allow information distribution.

To further promote communication and information sharing, the following website (http://www. wegenresearch.wales) and twitter account (@WeGeNresearch) have been created.

\section{Data handling within a WeGeN project}

Data collection spreadsheets are produced and distributed to each site lead by the project lead prior to initiating data collection. Each site lead collates the local data and the returns the file to the project lead in an anonymised format.

Each site lead maintains the right to interpret the data for their own site and are encouraged by WeGeN to present in local or regional forums. WeGeN suggests that presentation of data at national events is by the project lead.

\section{Authorship}

Authorship is on a named author basis for all predetermined lead researcher(s) for each project. These researchers are those who conceived, developed, delivered the project and wrote the manuscript. All other contributors, predominantly those that collected the data, are listed as collaborators.

Each project will be required to reference the research network and all collaborators using the phrase 'on behalf of the WeGeN network' at the end of the authorship listing. This model of authorship is based on contribution and premised on the International Committee of Medical Journal Editors. Recommendations for the Conduct, Reporting, Editing, and Publication of Scholarly Work in Medical Journals. ${ }^{15}$

\section{Findings to date}

There are four projects in progress using the WeGeN network, all of which anticipate peer review publication on maturity of the data.

The first is a multicentre audit of the British Geriatrics Society Silver Book Guidelines ${ }^{16}$ concerning the care of older persons within the first 24 hours in hospital. This was solely performed through WeGeN from the point of inception to delivery. After reviewing the Silver Book standards, data were collated simultaneously throughout December 2015, with 9 participating sites in South Wales providing data sets representing 354 patients.

The second project is a multicentre audit of the care of patients diagnosed with delirium. WeGeN facilitated data collection at eight Welsh sites. Six returning sites have performed preliminary analysis of their data and are addressing local areas for improvement while the overall collation of results is ongoing.

Our third project concerns the care of acutely unwell patients with a diagnosis of Parkinson's disease admitted to hospital. Data collection is still ongoing as a collaboration between the Welsh Parkinson's Disease Network and WeGeN to maximise the amount of sites involved and data collected.

The final ongoing project is a data collection focusing on the older emergency surgical patient. As part of a larger annual UK and Belgian data collection (http:// www.opsoc.eu), WeGeN has facilitated the addition of a further site (Abergavenny) to the established Cardiff OPSOC site and will coordinate Welsh data collection for this audit.

\section{NETWORK EVOLUTION AND FUTURE PLANS}

The use of a collaborative network carries inherent limitations when using observational data, such as a lack of a comparator group, variations in data collection between sites and ensuring uniform training for measures that require accuracy and reliable reproducibility. To mitigate this, our initial projects are comparatively small and led by selected members of WeGeN, or embedded within other existing data collection collaborations, such as the Welsh Parkinson's Disease Network and OPSOC.

It is anticipated that as the network matures and gains experience, the network lead will actively guide project leads during protocol development. This will help focus data collection towards outcome measures that are validated, simple, objective and reproducible; such as 30-day mortality or readmission to hospital.

We anticipate a 'learning curve' and it is likely that the structure, advice and leadership delivered by the WeGeN team will evolve. Given the peripatetic nature of trainee doctors, an essential part of the maturing process will be the internal development of future network leads who will have previously been site leads, then project leads, thus promoting those actively experienced with WeGeN organisation and process and preserving knowledge gained.

Beyond the cohorts which we describe here, we are anticipating further data collection in an ongoing manner. We have and will continue to advertise and promote WeGeN. Specific data collections will depend 
on the interests of any collaborator who wishes to use the network.

\section{COLLABORATION}

A prerequisite of using WeGeN will be the requirement of each project lead to securely retain collected data for at least 5 years. Therefore, each WeGeN project will be amenable and receptive to data sharing and further analyses of data with any researcher who can generate novel results using collected data. WeGeN is also receptive to the construction of other UK registrar-led research collaboratives. Furthermore, WeGeN would actively welcome involvement in the formation of combined regional platforms, or supporting the development of national, European or International platforms in a similar fashion to our surgical colleagues. 5811

\section{FURTHER DETAILS}

WeGeN has received no external funding and is being operated within the Supported Professional Activity sessions that the trainees have within their job plans. This arrangement has been supported by the Training Programme Director for Geriatric Medicine in South Wales. In the future as the Network grows, we hope to finance the network through external grant income.

\section{Twitter Follow Benjamin Jelley @WeGeNresearch}

Collaborators Joel Abbott; Adam Aboalkaz; Shridhar Aithal; Camilla Baker; Ruth Bevan; Faisal Bukhari; Michael Bull; Tamar Collier; Charles D'Souza; Glesni Davies; Kathryn Davies; Karl Davis; Lauren Evans; Swapna Fernandez; Amy Ferris; Triveni Gagan; Avril Gibbons; Andrew Haden; Wyn Harris; Thomas Hickin; Nia Humphry; Anthony James: Karina James; Amy Jones; Shoned Jones; Maiwish Khattack; Mukesh Kumar; Zena Marney; Robin Martin; Khun Maung; Biju Mohamed; Imran Mohamed; Bethan Morris; Rhian Morse; Praveen Pathmanaban; Elba Peter; Angela Puffett; Bella Richard; Elizabeth Robertson; Mathew Rogers; Rebecca Roper; Laura Rozier; Inderpal Singh; Thayapary Sivagnanam; Peter Slade; Zahid Subhan; Victoria E A Suter; Christopher Thomas; Sanjeev Vasishta; Ajit Verma; Allen Wilson.

Contributors $\mathrm{BJ}, \mathrm{SL}, \mathrm{JB}$ and $\mathrm{JH}$ contributed to the conception of this article. $\mathrm{BJ}$ and $\mathrm{JH}$ performed literature searches. BJ, SL, JB and $\mathrm{JH}$ drafted the article and revised it critically for important intellectual content. BJ, SL, JB and JH provided final approval of the version to be published. BJ acts as the guarantor.

Competing interests None declared.

Provenance and peer review Not commissioned; externally peer reviewed.
Data sharing statement This protocol article contains reference to four ongoing projects that have not been published, but are not directly related to the content of this manuscript.

Open Access This is an Open Access article distributed in accordance with the Creative Commons Attribution Non Commercial (CC BY-NC 4.0) license, which permits others to distribute, remix, adapt, build upon this work noncommercially, and license their derivative works on different terms, provided the original work is properly cited and the use is non-commercial. See: http:// creativecommons.org/licenses/by-nc/4.0/

\section{REFERENCES}

1. Joint Royal Colleges of Physicians Training Board. Speciality training curriculum for geriatric medicine. London, 2013:1-120.

2. Assiotis A, Azegami S, Pengas I, et al. Tips for success in interviewing for a national training number in trauma and orthopaedics. Bull R Coll Surg Engl 2015;97:E30-3.

3. Ellis MC, Dhungel B, Weerasinghe $\mathrm{R}$, et al. Trends in research time, fellowship training, and practice patterns among general surgery graduates. J Surg Educ 2011;68:309-12.

4. Brubaker L, Kenton K. Clinical research education study teams: a research curriculum for obstetric and gynecology residents. Obstet Gynecol 2011;117:1403-7.

5. Dowswell G, Bartlett DC, Futaba K, et al. How to set up and manage a trainee-led research collaborative. BMC Med Educ 2014;14:94.

6. Gaglioti AH, Werner JJ, Rust G, et al. Practice-based Research Networks (PBRNs) Bridging the Gaps between Communities, Funders, and Policymakers. J Am Board Fam Med 2016;29:630-5.

7. Spears W, Tsoh JY, Potter MB, et al. Use of community engagement strategies to increase research participation in practice-based research networks (PBRNs). J Am Board Fam Med 2014;27:763-71.

8. Eurosurg Collaborative. EuroSurg: a new European student-driven research network in surgery. Color Dis 2016;18:214-15.

9. Gheorghe A, Roberts TE, Pinkney TD, et al. The cost-effectiveness of wound-edge protection devices compared to standard care in reducing surgical site infection after laparotomy: an economic evaluation alongside the ROSSINI trial. PLOS ONE 2014;9:e95595.

10. Pinkney TD, Calvert M, Bartlett DC, et al. Impact of wound edge protection devices on surgical site infection after laparotomy: multicentre randomised controlled trial (ROSSINI Trial). BMJ 2013;347:f4305.

11. GlobalSurg Collaborative. Mortality of emergency abdominal surgery in high-, middle- and low-income countries. Br J Surg 2016;103:971-88.

12. PLOS Medicine Editors. Observational studies: getting clear about transparency. PLoS Med 2014;11:e1001711.

13. Peat G, Riley RD, Croft $P$, et al. Improving the transparency of prognosis research: the role of reporting, data sharing, registration, and protocols. PLoS Med 2014;11:e1001671.

14. Slack. Slack: be less busy [Internet]. [cited 31 October 2016] https://slack.com/

15. International Committee of Medical Journal Editors. Recommendations for the Conduct, Reporting, Editing, and Publication of Scholarly Work in Medical Journals. ICMJE, 2015. http://www.icmje.org/icmje-recommendations.pdf

16. Banerjee J, Conroy S, O'Leary V, et al. Silver Book: Quality Care For Older People With Urgent \& Emergency Care Needs. The British Geriatric Society, 2012. 